\title{
EVALUACIÓN DE LA FORMACIÓN Y ACTIVACIÓN DEL CAPITAL SOCIAL FRENTE A LA APLICACIÓN DE UNA POLÍTICA PÚBLICA \\ Caso: Programa EMPRENDE y su desarrollo durante el período 2005-2010 en la Mesa Público Privada de Turismo de Panguipulli.
}

\author{
$M^{a}$ Cecilia Silva Pineda \\ Universidad Catolica de Temuco \\ Chile \\ silvapineda.c@gmail.com \\ Francisco Gatica $\mathbf{N}$ \\ Universidad del Bio Bio \\ Chile \\ fgatica@ubiobio.cl
}

\section{RESUMEN.}

Esta investigación busca conocer el comportamiento de los actores turísticos en un territorio y las relaciones que se generan entre ellos y con otros actores extraterritoriales producto de la aplicación de una Política Pública, ya que estos “nexos” son un elemento básico al momento de desarrollar la gestión de cualquier destino turístico, por la importancia al momento de generar estrategias y acciones las cuales deben promoverse para adaptarse a las nuevas condiciones del contexto. El estudio se centra en el desarrollo del Programa Emprende en el territorio comunal durante el período en el cual estuvo en ejecución, esto es desde el año 2005 hasta el 2010, momento en el cual se decide dar por finalizado el Programa a nivel nacional. Para este estudio se utiliza el análisis longitudinal de Redes, a partir de la realización de entrevistas y análisis de las actas de reuniones, empleando algunas definiciones conceptuales contenidas en el programa UCINET, el cual permite visualizar los grafos y generar diferentes indicadores de variedad y riqueza estructural.

Palabras claves: redes sociales, políticas públicas, turismo. 


\title{
SOCIAL CAPITAL FORMATION AND ACTIVATION EVALUATION IN FACE OF THE APPLICATION OF PUBLIC POLICY \\ Case: EMPRENDE Program and its development during 2005-2010 at Panguipulli’s Private-Public Tourism Board.
}

\author{
$M^{\text {a }}$ Cecilia Silva Pineda \\ Universidad Catolica de Temuco \\ silvapineda.c@gmail.com \\ Francisco Gatica N \\ Universidad del Bio Bio \\ fgatica@ubiobio.cl
}

\begin{abstract}
.
This research is intended to get to know the behaviour of different people who work in the tourist field in a specific area and the connections that emerge among them, as a result of a Public Policy, and the relationship with other people who come from different parts of the region. These connections are a fundamental element when dealing with the management of any tourist area given the importance of creating strategies and actions which must be directed to adapt to the new conditions of the context. The research focuses on the development of the Emprende Program in Panguipulli from 2005 to 2010, year in which the Program ended throughout the country. This study was based on a longitudinal analysis of networks and it was carried out through interviews and analysis of meeting records, using some conceptual definitions from the UCINET program, which allows the visualization of graphs and the creation of different indicators of structural variety and richness.
\end{abstract}

Key words: social networks, public policies, tourism 


\section{INTRODUCCIÓN.}

La asociatividad es uno de los elementos constitutivos del Capital Social, principalmente porque las distintas formas de asociatividad dan cuenta de experiencias en las cuales se fomentan elementos como la confianza social, la adhesión a normas y la reciprocidad. Cobra una importancia central en la promoción de estas formas de asociatividad las Políticas Públicas. No basta con aumentar las capacidades personales de los individuos si no hay un conjunto de redes sociales que hagan que esas capacidades puedan efectivamente ponerse en juego.

Hoy podemos observar que en diversas coyunturas se repite la misma reacción frente a la emergencia, respecto a diferentes demandas de la ciudadanía. Un claro ejemplo de esto, son las recientes manifestaciones a lo largo de Chile, en donde queda explícito que las personas, a través de acciones colectivas, son capaces de enfrentar sus cuestionamientos a las políticas generadas desde el Estado, las que en algunos casos no apuntan a revertir requerimientos de la sociedad en su conjunto. En su mayoría se han plateado demandas que carecen de canales reales de participación y, por lo tanto, se realizan fuera de cualquier ámbito institucional. Hoy la acción colectiva tiene que ver básicamente con redes de vínculos asociativos, sociabilidad, confianza, cooperación y reciprocidad.

Frente a este escenario surge entonces el siguiente cuestionamiento ¿Por qué evaluar las políticas, programas y proyectos públicos a la luz de las redes sociales? Algunas de las alternativas son:

- Por una mayor demanda de participación y transparencia por parte de la sociedad

- Ciudadanía cada vez más exigente con los servicios públicos y con respuestas sostenibles

- Necesidad de ajustes y correcciones fiscales dentro de un marco de modernización y racionalización de la gestión pública

- Costo político por la mala gestión de Programas Públicos

- Necesidad de mayor participación en las políticas públicas ya sea en el diseño, ejecución y control.

- La presencia de redes asegura una mayor probabilidad de externalidades y procesos de aprendizaje alrededor de las políticas públicas. 
La nueva Gestión Pública exige sistemas de evaluación, siendo los problemas más comunes en estos sistemas: la planificación, los planes de desarrollo, el presupuesto, la información y evaluación. Entonces, la evaluación de Políticas Públicas es clave para asegurar que la gestión pública sea eficiente. En este sentido Chile cuenta con algunos sistemas de evaluación de Políticas Públicas: en el 2008 se realizó la evaluación de ocho Políticas Públicas y otras ocho se plantearon para el 2009.

A raíz de este planteamiento surgen entonces algunos supuestos que pudieran plantearse ¿Qué tan efectiva es la aplicación de Políticas Públicas en ciertos territorios? ¿Qué tan cierta es la relación público- privada que se genera producto de estas intervenciones? ¿Es posible gestionar de manera más eficiente las relaciones entre los diversos agentes locales que impulsan y desarrollan la actividad turística local?

Esta investigación se basa en la existencia de una relación directa entre la dinámica relacional que se produce entre los agentes implicados en el desarrollo turístico en la comuna de Panguipulli y el nivel que alcanza este desarrollo. Este estudio se aplica a la Mesa público privada de turismo, ya que es una de las líneas de trabajo que se priorizó a través del Programa Emprende, siendo una de las principales actividades económicas que se desarrollan en la comuna y a la existencia, antes de la implementación del Programa, de dicha Mesa.

\section{Desarrollo local y la importancia en la participación de actores.}

Sergio Boisier (2003) plantea que el desarrollo local, en muchos casos, es el fruto de la intervención de diferentes actores sociales que forman parte de una población que convive en un territorio concreto. Tal interacción crea un valor compartido a modo de capital social por todos y cada uno de los agentes sentando de esta forma los cimientos para el desarrollo local. En el ámbito actual, cabría diferenciar cuatro tipos de actores: Públicos, Privados, las organizaciones del tercer sector y los Sectores Sociales no organizados. Las relaciones que se configuren entre ellos son las relaciones que van a determinar el desarrollo local.

\section{Análisis de Redes Sociales.}

El trabajo en Red ayuda a que una comunidad actúe más coherente con la realidad, 
permite canalizar de cierta forma las necesidades más significativas de cada individuo y logra detectar intereses colectivos que permitan generar actitudes de acción conjunta dentro de una comunidad. Las posibles interacciones entre individuos y organizaciones en una Red Social además de representar flujos, podrían generar un impacto relevante en el comportamiento y proceder de los actores, en el proceso de convivencia de la Red y en los resultados de dicha interacción (Lozares, 1995).Es importante representar gráficamente la forma de la Red de actores, así como poder medir y establecer índices algebraicos que representen las propiedades de las estructura en análisis. En este sentido, se pueden distinguir dos aspectos en el esfuerzo por medir la estructura y organización de la Redes: primero, la estructura general de la Red y el nivel de integración que alcanza (visión sociocentrica), y segundo, la posición que cada uno de los actores ocupa en el conjunto de la Red (egonets).

Una red social territorial de tipo asociativa puede tener distintos niveles de asociación; existirán redes con objetivos muy acotados, mientras que otras asociaciones más avanzadas podrían considerar desarrollar acciones conjuntas entre distintos actores de la red que pueden responder a diferentes lógicas y pueden estar o no en el territorio. En la medida en que la red avanza hacia niveles superiores de asociación, los vínculos se fortalecen y se producen entornos virtuosos de desarrollo.

En relación a los contenidos de una Red, uno de los elementos primordiales es el grado de consolidación de la red, pudiendo hacer una distinción entre redes menos consolidadas o primarias y otras consolidadas o avanzadas. Este grado de consolidación tiene que ver, con la fortaleza del tipo de relación o vínculo que se produce entre los actores o nodos de la red. Mientras más fuerte, mayor número de emprendimientos conjuntos y cooperación en la Red. La meta para los territorios es que existan cada vez más redes avanzadas o consolidadas, ya que su funcionamiento supone la existencia de procesos de desarrollo localizados. En este sentido interesa no sólo la mayor cantidad de actores presente en una red articulada por una política pública, sino que también se requiere un mayor nivel de vinculación y una mayor variedad de funciones y competencias, siendo estos elementos claves para la innovación. La generación de redes equilibra los costos de transacción, lo que son producto de los niveles de desconfianza, y de las competencias tecnológicas (Williamson, 1985). 


\section{Políticas Públicas.}

Parte fundamental del quehacer del Gobierno se refiere al diseño, gestión y evaluación de las Políticas Públicas (Lahera, 2004). Una política pública, como plantea Podestá (2001), es un instrumento de trabajo mediante el cual se pretende alcanzar desde el Estado, en forma sistemática y coherente, ciertos objetivos de interés para el bienestar de toda la sociedad civil. Son cursos de acción destinados a la solución de problemas, donde inclusive el no hacer nada es una acción que se tiene que tomar en consideración y poner en práctica o no. Una Política Pública es un conjunto de acciones intencionales y causales, orientadas a la realización de un objetivo de interés/beneficio público, cuyos lineamientos y recursos se reproducen en el tiempo de manera constante y coherente, en correspondencia con el cumplimiento de funciones públicas que son de naturaleza permanente o con la atención de problemas públicos cuya solución implica una acción sostenida (Aguilar, 2009).

Para el desarrollo de Políticas Públicas, o intervenciones estatales, en un sentido amplio, el Estado cuenta con variadas formas de acción. De este modo, el Estado podrá intervenir mediante transferencias al sector privado, definición de normas, aplicación de programas, incentivos y franquicias tributarias, desarrollo directo de obras, provisión de servicios, corrección de desequilibrios, etc. En este sentido Ferreiro (2009) plantea que las Políticas Públicas son desarrolladas por diversas instituciones que cuentan con diferentes instrumentos para realizarlas y que en su aplicación ejercen influencia en distintos aspectos de la vida de los ciudadanos. Dado que toda Política Pública está llamada a incidir positivamente sobre el bienestar social, por un lado, y supone, por otro la asignación de recursos con altos costos de oportunidad, es esencial que las intervenciones del Estado se sometan a evaluaciones rigurosas. Así, habrá programas o políticas que apunten preferentemente a mejorar la competitividad de la economía, la equidad social, el fomento de determinadas actividades económicas, la focalización de los subsidios, la descentralización, equidad de género, etc. Sin embargo, cualquiera sea el objetivo o valor social que una política persiga, este debiera ser explicitado y medido. Ya no basta una racionalidad instrumental, sino que el Estado, junto con otros actores debe intervenir con una lógica sistémica generadoras de entornos competitivos.

Una Política Pública debe estar sustentada en una ideología, que oriente los cursos de acción, así como la toma de decisiones, el establecimiento de prioridades y la 
asignación de recursos financieros en el ámbito público, que permita crear los canales adecuados para transmitir flujos de información entre el Estado y la sociedad civil, por último debe señalar los objetivos, recursos, señales, límites y plazos para el cumplimiento de las alianzas o acuerdos y llevar a la práctica la propuesta de trabajo (De la Maza, 2004). Un avance interesante y particularmente relevante en cuanto a las relaciones con los actores sociales es la introducción del enfoque de planificación territorial de las Políticas y Programas. Este permite coordinar en un territorio dado a instituciones y actores de diversa naturaleza y relacionarse de modo conjunto con la demanda de ese territorio.

El análisis de las políticas públicas requiere de un enfoque interdisciplinario desde el cual se explique la interacción entre las instituciones, los intereses y las ideas que conforman los procesos de las políticas. Definitivamente se requiere que un estado más pequeño, pero más fuerte (F. Fukuyama 2005).

Entonces, la importancia de desarrollar diversas metodologías de análisis de las políticas públicas y los resultados de las mismas en los territorios, es clave al momento de considerar la aplicación de éstas en diversas instancias locales, en especial ya que son estas mismas quienes se verán beneficiadas o no de los resultados que pudieran generarse, y por consiguiente, de los efectos que pudiera tener en las redes locales de trabajo.

\section{Programa Chile Emprende.}

El Programa Chile Emprende nace por la necesidad de introducir cambios en las Políticas y Programas Públicos de apoyo a las empresas de menor tamaño y su entorno territorial a través de la descentralización, gobernanza y competitividad (Orellana, 2006). El Programa Chile Emprende se enmarcó en los compromisos del Gobierno del Presidente Lagos, los cuales se encuentran incluidos en su programa "Para crecer con igualdad" del período de 2000 - 2006. Desde el punto de vista político, la propuesta de este programa, apunta a lograr un desarrollo territorial equilibrado del país y fortalecer a la micro y pequeña empresa, las que generan más de dos tercios de los empleos, siendo claves en el desarrollo de oportunidades económicas, de mejoramiento de los ingresos y del empleo. Con esto, se buscó promover los tejidos productivos territoriales, integrados mayoritariamente por empresas de menor tamaño, para que conformen un sector dinámico de la economía que aporte significativamente al aumento de la productividad, a la creación de mejores empleos, al desarrollo social y a la superación de la pobreza. Además de 
promover la cooperación entre empresarios y la conexión de estos con los mercados, sobre la base de una gestión Público-Privada (Orellana, 2006).

El Programa buscaba fortalecer la asociatividad gremial de los empresarios y a la promoción de la cooperación entre el sector público y privado para el diseño de estrategias de desarrollo territorial. El enfoque de este Programa exigió nuevas formas de coordinación de la institucionalidad de fomento en los diferentes niveles de la administración (local, territorial, regional y nacional) y a esquemas flexibles y más eficaces de relación entre empresas y entre el mundo público y el privado, procurando generar las economías de aglomeración que mejoren la competitividad del territorio, corrigiendo una debilidad estructural de las políticas públicas en un actuar sectorial, carente de una visión transversal, que es lo que obliga una mirada más territorial y menos sectorial de los problemas.

Las políticas rara vez se extinguen por completo, es más habitual que cambien o se combinen con otras. Sin embargo, existen políticas con aspectos temporales definidos, que dejan de existir por diseño. Es el caso del Programa Emprende el cual deja de desarrollarse a mediados del año 2010, producto de una modificación a las Políticas de Fomento en los servicios dependientes del Gobierno. De aquí que se considera que la experiencia de este Programa resulta interesante de analizar, dado que tiene un tiempo acotado, permitiendo hacer una evaluación ex-ante y ex-post de la política pública, lo que es un ejercicio clave para el aprendizaje y evolución de la intervención del Estado.

Entonces podemos saber de forma teórica el funcionamiento de las estructuras sociales, pero ¿dicho funcionamiento es el mismo cuando uno ve esas estructuras sociales bajo una perspectiva concreta? ¿Sabemos como interaccionan estos actores entre sí? ¿La generación de Políticas Públicas a corto plazo genera efectos en los territorios y en sus actores? ¿Queda en los territorios un capital social derivado de la aplicación de la política pública?

\section{Caso de estudio: Mesa Público Privada de Turismo de Panguipulli.}

Producto de una demanda de diversas organizaciones turísticas locales, y en conjunto con el Departamento de Turismo de la Municipalidad de Panguipulli, en el año 2000 surge la Mesa de Turismo público-privada de la comuna de Panguipulli. Esta 
instancia nace con la finalidad de coordinar a los diversos actores de la actividad turística a nivel comunal. Este trabajo en conjunto ha permitido gestionar una serie de acciones tendientes a mejorar las condiciones del territorio y de los empresarios. Según consta en las diversas actas de reuniones de la Mesa de Turismo, en sus inicios esta mesa contó con la participación de cuatro organizaciones turísticas, las cuales representaban a 63 empresas del rubro. En relación a la intervención de los servicios públicos en el territorio, éstas se desarrollaban a nivel individual o a través de una organización específica. Los principales apoyos provenían de SERCOTEC, FOSIS e INDAP. Estos servicios tenían una alta intervención en el territorio, debido a las características de los usuarios y los instrumentos con que dichos servicios contaban.

En el año 2005, y producto de la implementación del Programa Emprende Chile (que más tarde pasaría a llamarse Chile Emprende), es que la Dirección regional de SERCOTEC propone como territorio piloto para la implementación del Programa a la comuna de Panguipulli, en especial por la existencia de un grupo de trabajo consolidado y a la representatividad que dicha agrupación simbolizaba para el territorio. Al momento de la implementación del Programa, la Mesa Público Privada de Turismo estaba compuesta por seis organizaciones, con un total de 117 empresarios (lo que representa un incremento del 85\%). Producto de esta misma implementación, SERCOTEC, SERNATUR, INDAP, FOSIS y SENCE, comenzaron a priorizar el territorio y la aplicación de sus instrumentos en los empresarios locales. Además se incorporaron a la Mesa de trabajo CORFO y CONAF. De esta manera se oficializó la constitución de la Mesa de turismo Sietelagos Emprende, la cual se encontraba compuesta por siete servicios públicos (SERCOTEC, SERNATUR, FOSIS, SENCE, INDAP, CORFO, CONAF), seis representantes privados (Presidentes de las organizaciones) y la Municipalidad de Panguipulli. Esta Mesa de trabajo comienza a concretar una serie de iniciativas en conjunto, bajo la lógica de trabajo del Programa Emprende (aporte en partes iguales entre privados, servicios públicos y Emprende). Algunos de los logros alcanzados más destacados son: participación en ferias de turismo nacionales e internacionales, capacitación constante de los empresarios en diversas temáticas, inversión pública en infraestructura local, fortalecimiento de la Mesa de Turismo, acceso a fuentes de financiamiento de proyectos, entre otros.

La implementación del Programa en la comuna permitió que otras organizaciones accedieran al trabajo de la Mesa de Turismo. En el año 2007 participaban once organizaciones comunales, las cuales representaban a un total de 192 empresarios 
de diversas áreas: turismo aventura, artesanía, turismo rural, alojamientos, alimentación y termas. Este mismo año, la Mesa de Turismo Sietelagos Emprende recibe un reconocimiento a nivel nacional por el trabajo y los logros obtenidos. La inversión lograda en el territorio hasta ese entonces, sobrepasó los $\$ 900.000 .000$ pesos chilenos.

En el año 2010, producto de las nuevas políticas de Gobierno en apoyo a las micro, pequeñas y medianas empresas, se pone término al Programa Emprende a nivel nacional, y por ende en la comuna. Fruto de esto, surge entonces la incertidumbre en las mesas de trabajo territoriales, respecto a la continuidad de las instancias creadas.

El cuadro $\mathrm{N}^{\circ} 1$ muestra las organizaciones privadas de base participantes de la Mesa de Turismo durante el desarrollo del Programa Emprende en la comuna de Panguipulli. En él se aprecia que a lo largo de la aplicación de la política de Estado, hubo un flujo de entrada y salida de agrupaciones, motivadas principalmente por el interés en participar del Programa.

Cuadro $\mathrm{N}^{\circ} 1$. Organizaciones privadas participantes de la Mesa de Turismo de Panguipulli.

\begin{tabular}{|l|c|c|c|}
\hline \multirow{2}{*}{ Organización } & \multicolumn{3}{|l|}{ Año participación } \\
\cline { 2 - 4 } & 2005 & 2007 & 2010 \\
\hline Asociación de Turismo de Panguipulli & $\mathrm{X}$ & $\mathrm{X}$ & $\mathrm{X}$ \\
\hline Asociación de Turismo de Choshuenco & $\mathrm{X}$ & $\mathrm{X}$ & $\mathrm{X}$ \\
\hline Cámara de Turismo de Coñaripe & $\mathrm{X}$ & & $\mathrm{X}$ \\
\hline Agrupación Puwinkul de Liquiñe & $\mathrm{X}$ & & $\mathrm{X}$ \\
\hline Artesanos de Neltume & $\mathrm{X}$ & $\mathrm{X}$ & \\
\hline Agrupación Pillanlafquen & $\mathrm{X}$ & & \\
\hline Cámara de Turismo de Neltume & & $\mathrm{X}$ & $\mathrm{X}$ \\
\hline Agrupación Trayenco de Liquiñe & & $\mathrm{X}$ & $\mathrm{X}$ \\
\hline Cámara de Turismo de Panguipulli & & $\mathrm{X}$ & $\mathrm{X}$ \\
\hline Artesanos de Liquiñe & & $\mathrm{X}$ & \\
\hline Asociación de Turismo Aventura & & $\mathrm{X}$ & \\
\hline Agrupación de Pesca Choshuenco & & $\mathrm{X}$ & $\mathrm{X}$ \\
\hline Red de Turismo Rural & & $\mathrm{X}$ & $\mathrm{X}$ \\
\hline Agrupación Los Maitenes & & $\mathrm{X}$ & $\mathrm{X}$ \\
\hline Agrupación de empresarios termales & & & $\mathrm{X}$ \\
\hline
\end{tabular}

Fuente: elaboración propia, a partir de actas de reuniones de la Mesa de Turismo. 
El cuadro $\mathrm{N}^{\circ} 2$ muestra a los servicios públicos que intervinieron en el territorio durante el proceso del Programa Emprende. En la mesa de trabajo estaban presentes aquellos servicios públicos de fomento que, por estructura debían participar en el Programa (SERCOTEC, SERNATUR, SENCE, FOSIS, CORFO) a los cuales se sumaron servicios que, por la temática priorizada como actividad productiva del territorio, se incorporan con sus instrumentos de apoyo (INDAP, SERNAPESCA, CONAF), además de la Municipalidad de Panguipulli, como agente coordinador del Programa en el territorio.

Cuadro $N^{\circ}$ 2.Instituciones públicas participantes en el Programa Emprende en la comuna de Panguipulli.

\begin{tabular}{|l|c|c|c|}
\hline \multirow{2}{*}{ Institución } & \multicolumn{3}{|c|}{ Año participación } \\
\cline { 2 - 4 } & 2005 & 2007 & 2010 \\
\hline Municipalidad de Panguipulli & $\mathrm{X}$ & $\mathrm{X}$ & $\mathrm{X}$ \\
\hline SERCOTEC & $\mathrm{X}$ & $\mathrm{X}$ & $\mathrm{X}$ \\
\hline SERNATUR & $\mathrm{X}$ & $\mathrm{X}$ & $\mathrm{X}$ \\
\hline SENCE & $\mathrm{X}$ & $\mathrm{X}$ & $\mathrm{X}$ \\
\hline FOSIS & $\mathrm{X}$ & $\mathrm{X}$ & \\
\hline CORFO & $\mathrm{X}$ & $\mathrm{X}$ & $\mathrm{X}$ \\
\hline CONAF & $\mathrm{X}$ & $\mathrm{X}$ & \\
\hline INDAP & $\mathrm{X}$ & $\mathrm{X}$ & \\
\hline SERNAPESCA & & & $\mathrm{X}$ \\
\hline
\end{tabular}

Fuente: elaboración propia, a partir de actas de reuniones de la Mesa de Turismo.

La Mesa de Turismo está constituida por los Presidentes de las organizaciones de turismo, quienes representan en esta instancia, los requerimientos de cada asociación. Esta mesa consta de una directiva, compuesta por un Presidente, Vicepresidente, Secretario y Directores, quienes son elegidos en base a la propuesta de los mismos socios. Durante le desarrollo del Programa Emprende, se contó con una Gerencia técnica, además de la Secretaría técnica en manos del Departamento de turismo de la Municipalidad de Panguipulli. La periodicidad de las reuniones es mensual y en ellas se analizan temas, se toman decisiones y acuerdos atingentes a los proyectos con enfoque turístico, implementados a través del Programa Emprende. 


\section{Configuración y Análisis de la Red Local de Turismo.}

\section{Planteamiento metodológico.}

Este trabajo desarrolló, a través del Análisis de Redes Sociales, una visión del comportamiento del Capital social o actores locales, frente a la intervención de una Política Pública y su comportamiento frente a esta nueva dinámica de trabajo. Para lograr los objetivos planteados se utilizaron técnicas como sociogramas, para analizar las relaciones sociales específicas existentes en las organizaciones definidas, tratando desde allí interpretar la estructura de relaciones. Las Unidades de análisis están constituidas por las instituciones públicas de apoyo al desarrollo productivo del territorio comunal y las organizaciones turísticas. La técnica empleada para la recogida de la información es la aplicación de encuestas, en base a dos componentes: intercambio de información y flujos de cooperación (este último componente considera apoyo técnico y financiamiento, como aspectos de análisis). Se definieron estas dos líneas de análisis, ya que son objetivos paralelos que se pretenden alcanzar con la aplicación de diversas políticas públicas. Para el análisis de las redes, en esta investigación se ha utilizado el programa UCINET, software libre que permite la creación de matrices para el análisis de Redes e incorpora la representación gráfica de Net Draw para las presentaciones dinámicas. Junto con ello se analizan dos propiedades de la Red: Indicadores de Centralidad y Sub grupos en relación a las Redes de información y cooperación.

\section{Proceso de obtención de la información.}

Para realizar este trabajo, se llevó a cabo el siguiente proceso para la recopilación de la información requerida:

- En primera instancia se determinaron los actores del objeto del estudio, grupo compuesto por los presidentes y/o representantes legales de organizaciones de turismo, informantes clave y funcionarios públicos que participaron en cada etapa de desarrollo del Programa Emprende en la comuna de Panguipulli, quienes entregaron los insumos necesarios respecto al intercambio de flujo en los dos ámbitos de interés del estudio: información y cooperación.

- Luego de obtenidos los datos sobre las relaciones, se realizó su análisis, utilizando para 
ello dos técnicas. Primero, se aplicó la técnica del Análisis de Redes Sociales, a través del programa de tratamiento de datos relacionales UCINET 6, el cual permite identificar y determinar las características estructurales de las redes de actores. Sobre la base del método de redes completas, se obtuvo como resultado el grueso de datos relacionales necesarios para el análisis de la Red. Desde esta base, el programa permite elaborar una matriz de adyacencia, soporte matemático sobre el que se graba la información real de las relaciones entre todos los actores. Como resultado de la matriz de adyacencia se obtuvo una serie de indicadores de centralidad sobre la estructura formal de la Red.

- Con los antecedentes necesarios, se procedió a presentar la dinámica relacional, identificando a los actores y las relaciones que se establecen entre ellos en función de los proyectos, programas y actividades que se realizaron de manera vinculante entre los actores. En cada grafo, los nodos representan a los actores del sistema, mientras que las flechas indican entre cuales de ellos se producen interacciones o vínculos, en los ámbitos de cooperación e intercambio de información, para la ejecución de actuaciones relacionadas con el Programa.

Para este estudio, se han analizado dos propiedades de la Red de actores: Ratios de centralidad y Sub grupos que conforman una Red.

Metodológicamente compararemos las redes de actores en tres períodos: antes, durante y después del desarrollo del Programa Emprende en la comuna.

\section{Dinámica Relacional en el Ámbito de la Información.}

\section{Intercambio de información 2005.}

En la figura $\mathrm{N}^{\circ} 1$ se observa la Red de actores en torno al intercambio de información durante el año 2005.

- Se aprecia la existencia de cuatro subgrupos, con diversidad, tanto en la cantidad de actores y conexiones.

- El subgrupo uno, está compuesto por dos actores, quienes están unidos entre sí, ya que ambos actores se encuentran ligados a la temática de turismo rural. Se encuentran más 
bien aislados del centro neurálgico de la Red y unidos sólo a un subgrupo de la Red.

- El subgrupo dos está compuesto por un solo actor (CONAF) y unido únicamente al actor Municipalidad, que es a su vez el punto de corte, por lo cual se encuentra en una posición de alta fragilidad dentro de la Red, siendo este nodo su única conexión con el resto de la Red de trabajo.

- El subgrupo tres está conformado por dos actores unidos entre sí y con el centro de la Red. Se encuentran medianamente aislados, ya que tienen una baja cantidad de conexiones con otros actores, tanto públicos como privados.

- El subgrupo cuatro es el conjunto de nodos con mayor cantidad de actores y conexiones, contando con un total de nueve actores, de los cuales cinco son servicios públicos. El subgrupo se encuentra en torno a dos actores principales, SERCOTEC y Municipalidad, debido a la cantidad de conexiones que ambos servicios alcanzan dentro de la Red de trabajo.

En general se aprecia un grupo de actores más bien pequeño, pero cohesionado, unidos en torno a la entrega de información de proyectos o iniciativas grupales e individuales, lo cual permite que hayan pocos vacíos estructurales. Un aspecto a destacar tiene que ver con la centralidad de la red. Es evidente que el actor con la posición más central es el Municipio. Si bien se reconoce la existencia de, al menos, otros actores con una importante centralidad en la misma, lo cuales realizaban intervenciones en el territorio antes de la llegada del Programa Emprende, era el Municipio quien canalizaba gran parte de la información en torno a proyectos. 
Figura $\mathrm{N}^{\circ} 1$. Red de actores Programa Sietelagos Emprende. Intercambio de información período 2005

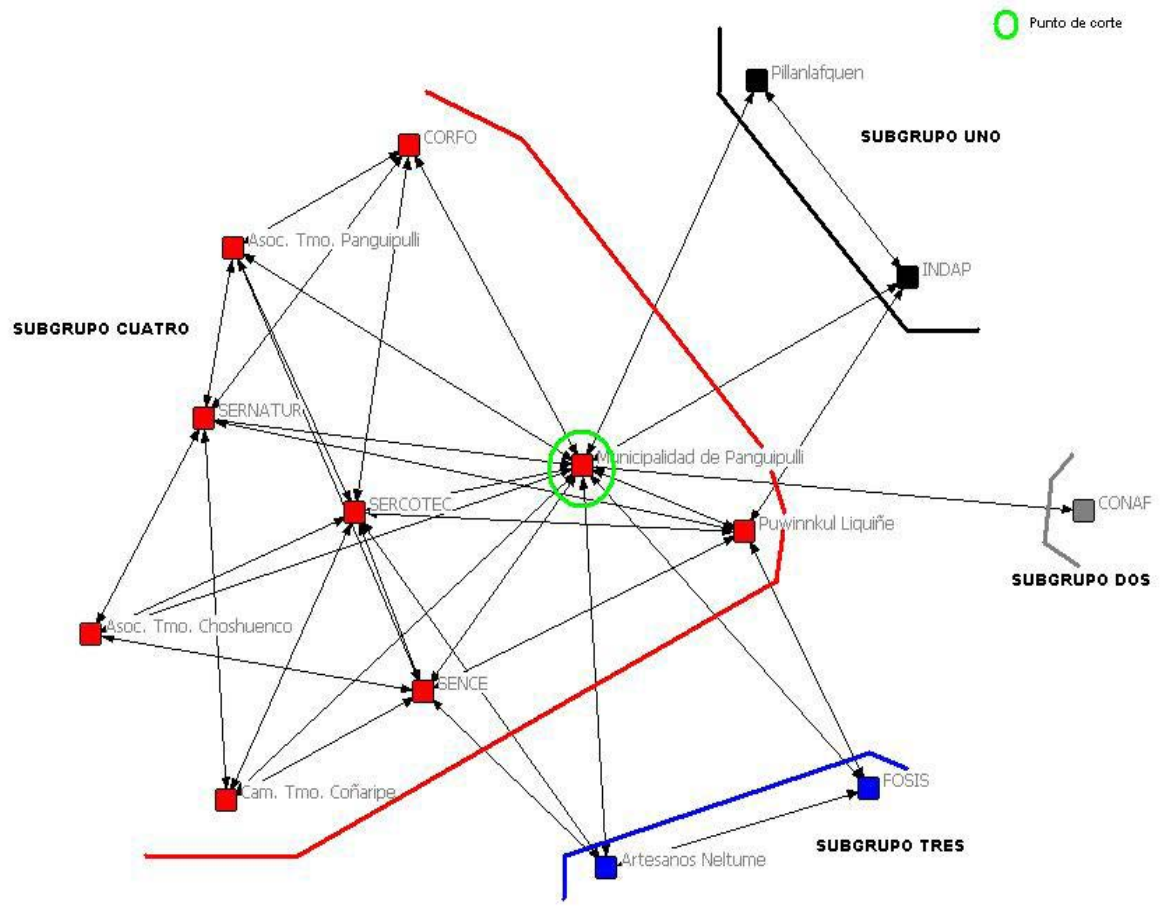

Fuente: elaboración propia.

Intercambio de información 2007.

La figura $\mathrm{N}^{\circ} 2$, muestra la configuración de la Red de actores durante el año 2007, en torno al intercambio de información.

- Esta Red está conformada por tres subgrupos de actores. En general se observa una mayor cantidad de actores y conexiones entre ellos, lo que muestra una Red más fortalecida y con mayores flujos de información.

- En el subgrupo uno se observan un total de cuatro actores, los cuales están, en su mayoría, ligados entre sí y con un promedio de cuatro conexiones. Tres de los actores 
están ligados a la temática del turismo rural.

- El subgrupo dos es el centro de la Red de información, ya que reúne actores con una gran cantidad de conexiones, entre sí y con otros actores de la Red. Esta compuesto por siete nodos, de los cuales cuatro son servicios públicos. Se observa un grupo más bien cohesionado y con altos flujos de intercambio de información.

- Finalmente, el subgrupo tres está formado principalmente por organizaciones privadas. Estos actores tienen un alto nivel de conexiones con el centro de la Red (en promedio seis conexiones), pero no se observa un gran número de conexiones entre los actores de este subgrupo.

Se observa que la intervención del Programa Emprende generó el ingreso y participación de una mayor cantidad de actores privados en la mesa de trabajo. Al haber un mayor número de actores, aumenta la relación entre los mismos, impulsando el intercambio en el flujo de información. Se aprecia además una consolidación en el intercambio de información, por lo cual no se observa vacío estructural ni punto de corte, lo que permite que los actores vean fortalecido su trabajo en el territorio. 
Figura $\mathrm{N}^{\circ} 2$. Red de actores Programa Sietelagos Emprende. Intercambio de información período 2007.

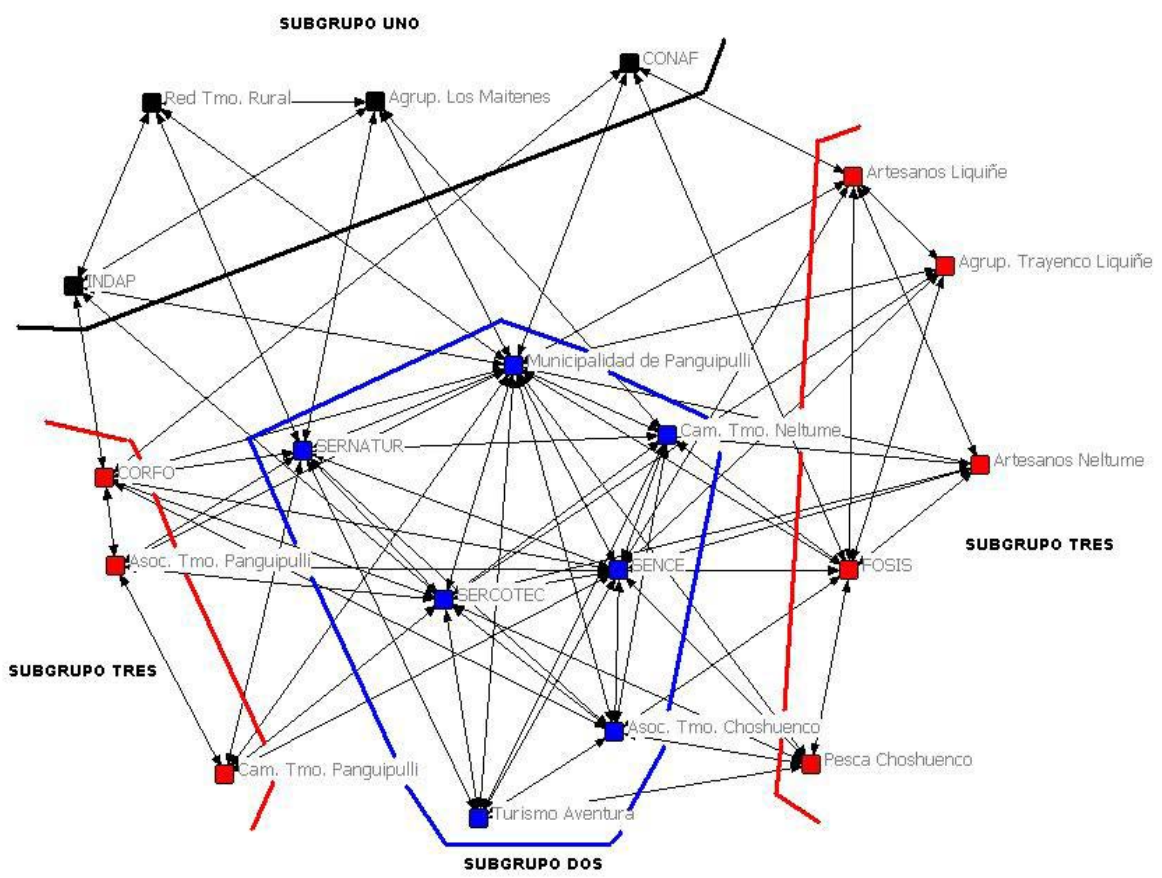

Fuente: elaboración propia.

Intercambio de información 2010.

En la figura $\mathrm{N}^{\circ} 3$, se visualiza la Red de actores en torno al intercambio de información una vez concluido el Programa Emprende en el territorio comunal:

- Esta Red esta compuesta por cinco subgrupos, lo que a primera instancia muestra una dispersión de los actores existentes.

- El subgrupo uno está compuesto por un solo nodo, con una gran cantidad de conexiones, pero más bien aislado del centro de la Red. Este nodo, está unido sólo a servicios públicos, lo que muestra una dependencia de los mismos, al momento de generar flujos 
de información.

- En el subgrupo dos, al igual que el subgrupo uno, sólo se observa un actor, el cual es a su vez el punto de corte de la Red, siendo la conexión entre el nodo Los Maitenes y el resto de la Red. El nodo del subgrupo dos está conectado al centro de la Red, pero sólo a servicios públicos.

- El tercer subgrupo lo compone una sola organización privada, conectada al centro de la Red por un actor, lo que muestra la alta fragilidad de éste dentro de la Red. No se observa conexión con ningún servicio público, por lo que necesita del actor del subgrupo dos para la obtención de información.

- El subgrupo cuatro lo componen tres actores, dos de ellos son organizaciones privadas, las cuales están unidas a los mismos actores dentro de la Red (SENCE y Municipalidad). Se observa en este subgrupo a SERNAPESCA, único actor público que ingresa a la Red de trabajo, una vez terminado en el territorio el Programa Emprende.

- Finalmente el subgrupo cinco, el cual está compuesto por los actores más cohesionados de la Red, debido al gran número de conexiones entre sí. Cuenta con el mismo número de actores público y privados.

Si bien se observa una menor cantidad de nodos y relaciones entre los mismos, existe un grupo de actores que logra mantener la conexión de trabajo posterior al término de la aplicación del Programa Emprende. Estos actores, están muy implicados en interacciones entre sí, lo que les proporciona una cohesión muy destacable a esta Red. 
Figura $\mathrm{N}^{\circ} 3$. Red de actores Programa Sietelagos Emprende. Intercambio de información período 2010.

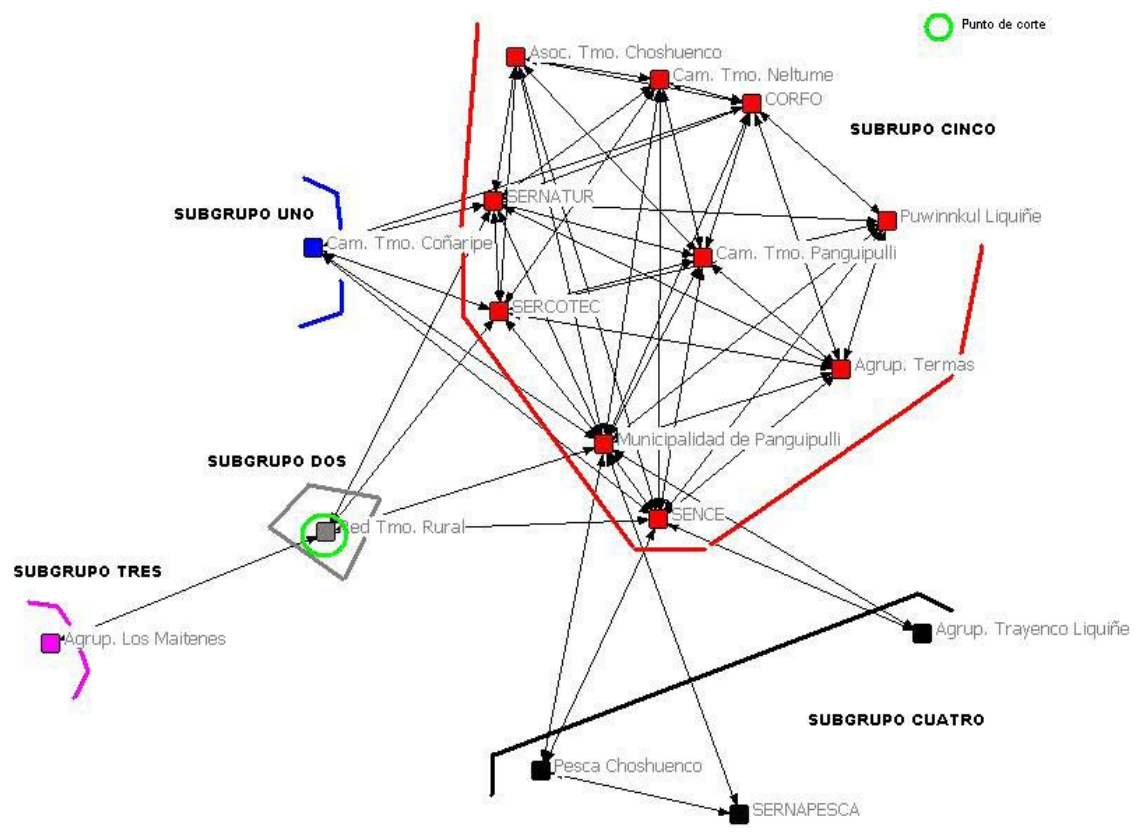

Fuente: elaboración propia

\section{Dinámica Relacional en el Ámbito de la Cooperación.}

\section{Intercambio de Cooperación 2005.}

En la figura $\mathrm{N}^{\circ} 4$, se muestra la Red de actores en torno a la cooperación antes de la llegada del Programa Emprende a la Mesa de trabajo de turismo de la comuna de Panguipulli.

- Se observa en primera instancia a un subgrupo uno, compuesto sólo por servicios públicos, asilados de la Red de actores en el ámbito de la cooperación. También se observa que no existía relación entre los nodos de este subgrupo.

- El subgrupo dos, en cambio, muestra al centro de la Red, compuesta por actores que desarrollan conexiones entre sus pares. Si bien sólo participan dos actores públicos 
(SERCOTEC y Municipalidad) son ellos los que concentran el mayor número de conexiones. Todas las organizaciones privadas de este subgrupo, se encuentran conectadas a los dos actores públicos, y por el contrario, no se observa conexión entre los organismos privados y entre los servicios públicos.

- En el subgrupo tres, sólo participa un actor, conectado al centro de la Red por un actor, lo que muestra la fragilidad de este subgrupo, debido a la dependencia de éste para el intercambio de cooperación.

En general en esta Red se observa una baja participación de actores privados y prácticamente nula intervención de actores públicos, recayendo sólo en dos servicios públicos la responsabilidad de entregar cooperación a los actores del sistema turístico de la comuna. La Municipalidad cumple un rol importante en esta Red, canalizador intervenciones en la comuna entre el sector público y privado. La densidad de la Red, es más bien baja, es decir, son muy pocos los actores que intervienen en ella y son también muy limitadas las relaciones que estos actores mantienen con sus pares. Sin embargo, resulta muy llamativo que la mayoría de los nodos se encuentran al margen de la Red, lo que probablemente tenga que ver con el hecho de que su línea de trabajo, no estaba dirigida necesariamente a iniciativas turísticas.

Figura $N^{\circ} 4$. Red de actores Programa Sietelagos Emprende. Cooperación período 2005.

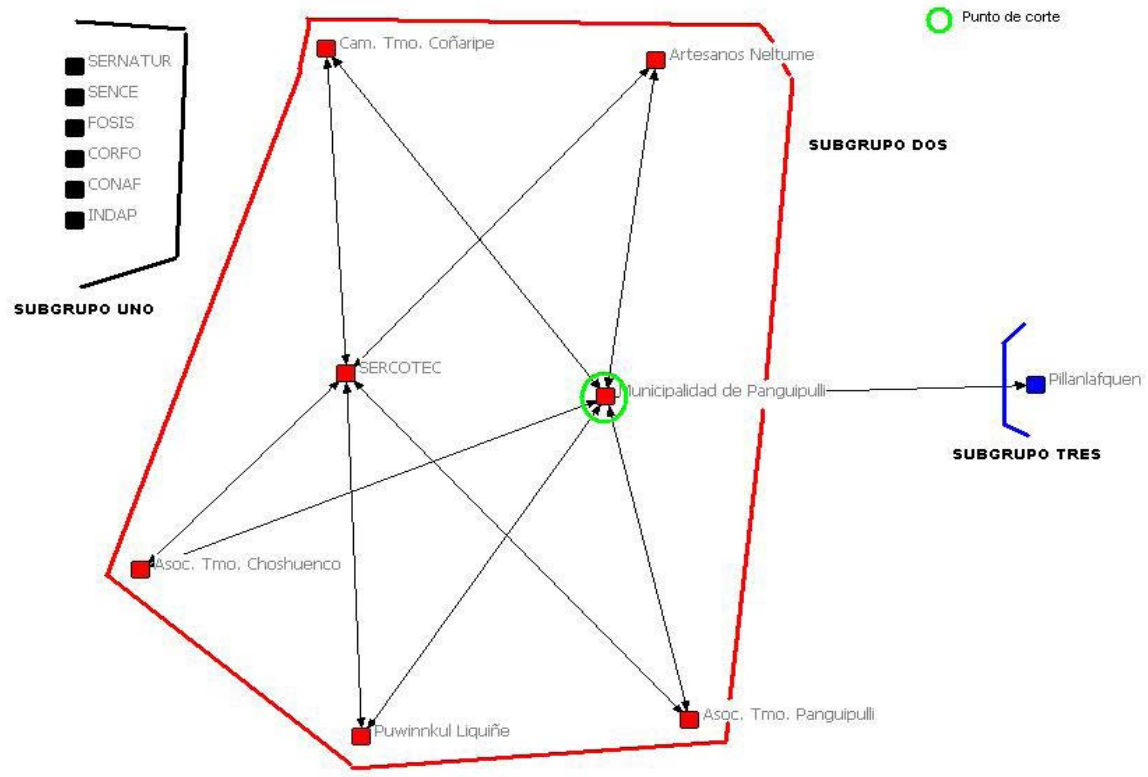

Fuente: elaboración propia. 


\section{Intercambio de Cooperación 2007.}

Se presenta en la figura $\mathrm{N}^{\circ} 5$, la Red de actores en torno al intercambio de cooperación existente el año 2007, durante la intervención en el territorio del Programa Emprende.

- El primer subgrupo está compuesto por cinco actores, dos servicios públicos y tres organizaciones privadas. Este subgrupo, que se observa en la configuración de la Red dividido en dos grupos no conexa entre sí, está medianamente vinculada al centro de la misma, con un promedio de tres conexiones por cada nodo. Las conexiones de estos actores son mayoritariamente con actores públicos de la Red.

- En el subgrupo dos, se encuentran los actores con más conexiones de la Red, lo cual lo transforma en el centro de la misma. Está compuesto principalmente por servicios públicos.

- El subgrupo tres, se muestra dividido, ya que no existe conexión entre dos nodos con sus pares pertenecientes a este subgrupo. Esta compuesto por siete actores, principalmente organizaciones privadas, con un promedio de cinco conexiones. Sus conexiones son mayormente con actores públicos del centro de la Red, además de haber una escasa conexión entre sus nodos.

En resumen, esta Red nos muestra una mayor participación de actores, en relación a la Red del año 2005, lo que permite una mayor cantidad de conexiones y por lo tanto mas interacción. No se observan vacíos ni punto de corte, muestra clara de una Red más fortalecida y una consolidación en la cooperación público-privada en torno a la actividad turística. Esta Red no tiene un único actor central, sino que la centralidad está compartida por un número importante de actores, lo que evidencia un claro multiliderazgo, que se manifiesta no sólo en el grado de centralidad, sino también en el de intermediación. Existe por tanto un conjunto de actores que muestran una cierta capacidad de liderazgo. Se observa finalmente una participación más directa de parte de los servicios públicos, ya que, producto de la intervención del Programa Emprende, deben orientan sus instrumentos y líneas de trabajo hacia la actividad turística. 
Figura N5. Red de actores Programa Sietelagos Emprende. Cooperación período 2007.

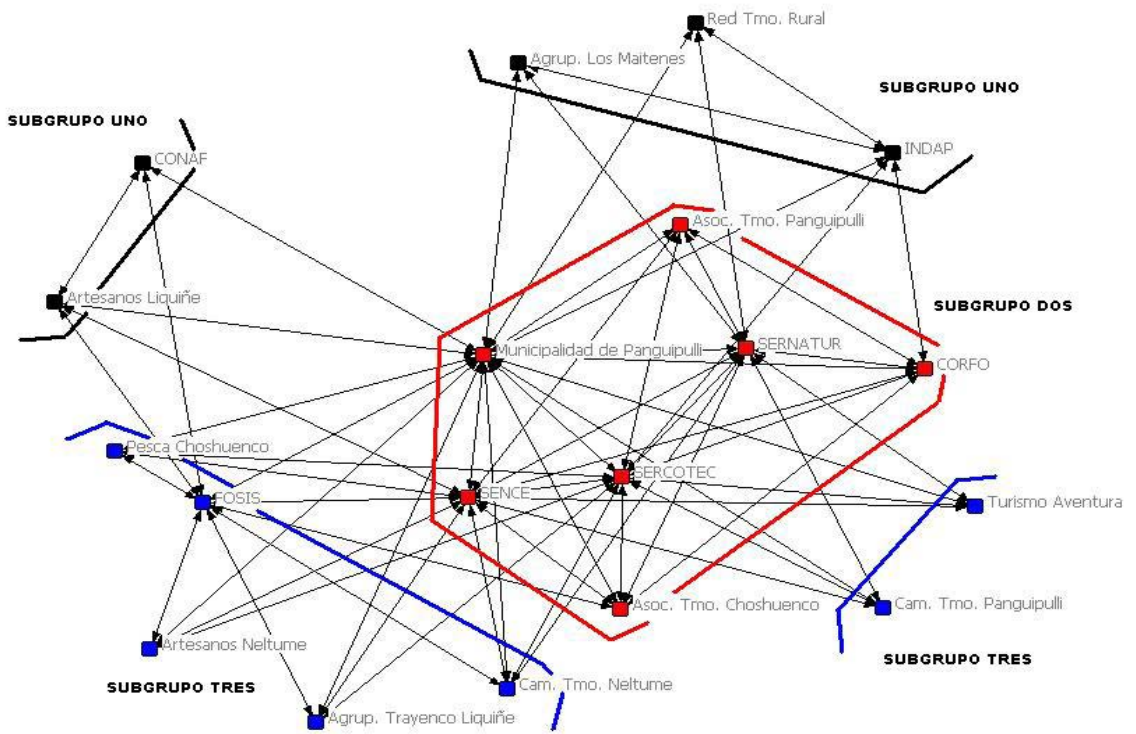

Fuente: elaboración propia.

\section{Intercambio de Cooperación 2010.}

La figura $\mathrm{N}^{\circ} 6$, nos muestra la Red de cooperación, una vez terminada la intervención del Programa Emprende en la comuna.

- El primer subgrupo está compuesto por un solo actor (Agrupación los Maitenes), la cual está aislada de la Red, ya que no tiene nexos con ningún otro actor, tanto público como privado.

- Un segundo subgrupo, está compuesto por un total de cinco actores. Cada uno de estos nodos, está unido al centro de la Red, a través de una sola conexión. Junto con esto, todos estos actores se encuentran unidos a nodos que son punto de corte, lo que muestra una posición de fragilidad dentro de la Red. Finalmente, se observa que no existe conexión entre los componentes de este subgrupo, por lo cual no hay cooperación entre ellos.

- En el tercer subgrupo se encuentran los actores con mayor cantidad de conexiones dentro de la Red durante este período. En él se hallan los tres actores que son punto de corte dentro de la Red (Cámara de turismo de Neltume, Asociación de turismo de 
Choshuenco, Municipalidad), de los cuales depende la interacción de otros nodos en el ámbito de la cooperación. En este subgrupo se encuentra el nodo con más conexiones (Municipalidad), lo que muestra el rol clave que juega una vez finalizada la intervención del Programa Emprende.

Esta Red, muestra una disminución en la participación de actores y en el número de conexiones entre sí, en relación a la Red del año 2007. A pesar de la disminución de una participación activa de diversos actores, destaca el ingreso de SERNAPESCA una vez finalizado el Programa Emprende en la comuna. La existencia de tres puntos de corte, es una clara señal de fragilidad de la misma. Se observa finalmente, como algunos actores públicos (SENCE, CORFO, SERCOTEC) se encuentran ligados a la Red, pero en una ubicación muy marginal de la misma, teniendo menor implicación en ella.

Figura $\mathrm{N}^{\circ}$ 6. Red de actores Programa Sietelagos Emprende. Cooperación período 2010

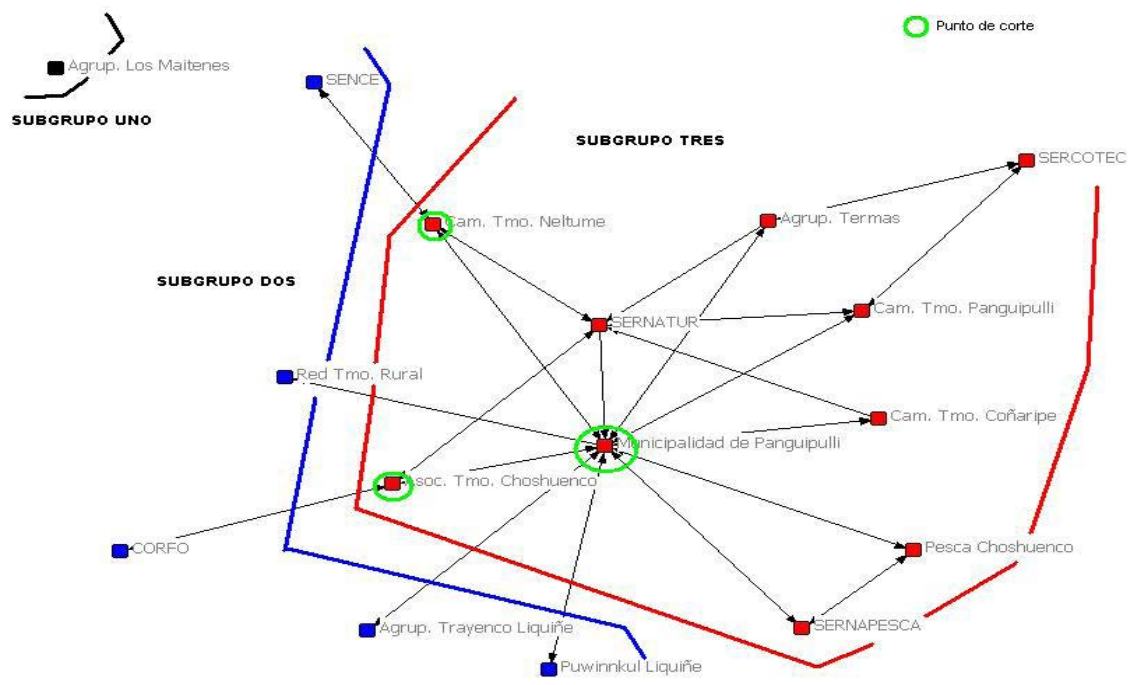

Fuente: elaboración propia

A continuación se presenta un cuadro resumen respecto al análisis de la Red de actores de la Mesa de Turismo. 
Cuadro $\mathrm{N}^{\circ} 3$. Resumen análisis Red de actores Programa Sietelagos Emprende. Intercambio de información período 2005-2007-2010.

\begin{tabular}{|l|c|c|c|}
\hline Ejes de análisis & 2005 & 2007 & 2010 \\
\hline No de actores de la Red $^{\text {o }}$ & 14 & 19 & 16 \\
\hline No de subgrupos de la Red $^{\text {Punto de corte }}$ & 4 & 3 & 5 \\
\hline Municipalidad de & No hay & $\begin{array}{l}\text { Red de Turismo } \\
\text { Rural }\end{array}$ \\
\hline
\end{tabular}

Fuente: elaboración propia.

Cuadro $\mathrm{N}^{\circ}$ 4. Resumen análisis Red de actores Programa Sietelagos Emprende. Cooperación período 2005-2007-2010.

\begin{tabular}{|c|c|c|c|}
\hline Ejes de análisis & 2005 & 2007 & 2010 \\
\hline $\begin{array}{l}\mathrm{N}^{0} \text { de actores de la } \\
\text { Red }\end{array}$ & 14 & 19 & 16 \\
\hline $\begin{array}{l}\mathrm{N}^{\mathrm{o}} \text { de subgrupos } \\
\text { de la Red }\end{array}$ & 3 & 3 & 3 \\
\hline Punto de corte & $\begin{array}{l}\text { Municipalidad de } \\
\text { Panguipulli }\end{array}$ & No hay & $\begin{array}{l}\text { Municipalidad de } \\
\text { Panguipulli, Cámara de } \\
\text { Turismo de Neltume, } \\
\text { Asoc. Turismo de } \\
\text { Choshuenco. }\end{array}$ \\
\hline
\end{tabular}

Fuente: elaboración propia.

\section{Análisis de Indicadores de la Red de Actores.}

Las Redes o Grafos son una importante herramienta al momento de representar interacciones entre individuos o grupos de individuos de manera ilustrativa. Sin embargo, estas no son la única herramienta de análisis. El Programa Ucinet permite, además de la visualización de la Redes, la aplicación de una serie de instrumentos matemáticos los cuales, a través de diversos indicadores, explican la estructura de una Red, dependiendo del análisis que se desee realizar.

Para este estudio se analizarán los Indicadores de Centralidad y Sub grupos, en relación a las Redes de información y cooperación. 


\section{Ratios de centralidad}

Permiten identificar a los actores más centrales, que por tanto tendrían un mayor nivel de poder en la red. Para esto se utilizan una serie de indicadores que entregan información específica sobre estas dimensiones. Para este estudio se han considerado tres factores: Degree, que permite conocer al actor que tiene mayor cantidad de relaciones dentro de la Red; Betweenness, que representa la posibilidad que tiene un nodo o actor para intermediar las comunicaciones entre pares de nodos, asumiendo un rol como actor "puente”; y Eigenvector, que permite conocer a el o los actores más centrales dentro de la estructura global de la Red considerando los contactos cercanos de cada nodo, así como una visión global de la red.

El cuadro $\mathrm{N}^{\circ} 5$ muestra los resultados de este análisis, donde destaca el rol central que cumple la Municipalidad de Panguipulli dentro de la Red analizada. Durante el tiempo de desarrollo del Programa Emprende en la comuna de Panguipulli, la Municipalidad fue el principal nexo entre el sector público y privado para el manejo y entrega de información. En el cuadro $\mathrm{N}^{\circ} 6$ se aprecia una constante en relación al rol de la Municipalidad como puente entre los diversos actores de la Mesa de trabajo al momento de cooperar en los diversos proyectos y programas desarrollados.

En el análisis de las relaciones (Degree) se aprecia que la Municipalidad es el actor que mayor cantidad de conexiones durante la duración del Programa Emprende, por ejemplo en los años 2005 y 2010, en el ámbito de la cooperación (cuadro $\mathrm{N}^{\circ} 6$ ) sostuvo relación con todos los actores.

Sobre el análisis de la intermediación (Betweenness) se puede concluir que es la Municipalidad quien cumple el rol de "puente" en la Red de actores, tanto en el ámbito del intercambio de información como cooperación.

Finalmente, en el análisis de la centralidad (Eigenvector) si bien la Municipalidad nuevamente cumple un rol protagónico, obteniendo los valores más altos en el ámbito del intercambio de información, destaca que dos actores (CONAF, Agrupación Los Maitenes) logran el mayor grado de centralidad en el ámbito de la cooperación en los años 2007 y 2010 respectivamente (cuadro $\mathrm{N}^{\circ} 6$ ). Para el caso de estos dos actores, si bien en el análisis de la Red de Cooperación ambos están situados lejos del centro de la Red, este índice nos muestra a aquellos actores que podrían conectarse de manera más directa con el centro de la Red y no requieren de intermediarios. 
Cuadro $\mathrm{N}^{\circ} 5$. Ratios de centralidad en torno al intercambio de información.

\begin{tabular}{|c|c|c|c|}
\hline $\begin{array}{c}\text { Ratios de } \\
\text { centralidad }\end{array}$ & Degree & Betweenness & Eigenvector \\
\hline 2005 & $\begin{array}{l}\text { Municipalidad } \\
13(1-14)\end{array}$ & $\begin{array}{l}\text { Municipalidad } \\
80.483 \text { (0 a } 80.483)\end{array}$ & Municipalidad 67,7\% \\
\hline 2007 & $\begin{array}{l}\text { Municipalidad } \\
18(4-19)\end{array}$ & $\begin{array}{l}\text { Municipalidad } \\
86.269 \text { (0 a } 86.269)\end{array}$ & $\begin{array}{l}\text { Municipalidad } \\
56 \%\end{array}$ \\
\hline 2010 & $\begin{array}{l}\text { Municipalidad } \\
14(1-16)\end{array}$ & $\begin{array}{l}\text { Municipalidad } \\
33.418 \text { (0 a 33.418) }\end{array}$ & $\begin{array}{l}\text { Municipalidad } \\
56.4 \%\end{array}$ \\
\hline
\end{tabular}

Fuente: elaboración propia.

Cuadro $\mathrm{N}^{\circ} 6$. Ratios de centralidad en torno al intercambio de cooperación.

\begin{tabular}{|c|l|l|ll|}
\hline $\begin{array}{c}\text { Ratios de } \\
\text { centralidad }\end{array}$ & Degree & Betweenness & Eigenvector \\
\hline 2005 & $\begin{array}{l}\text { Municipalidad } \\
6(0-6)\end{array}$ & $\begin{array}{l}\text { Municipalidad } \\
16(0-16)\end{array}$ & $\begin{array}{l}\text { Municipalidad } \\
74,1 \%(69.85 \%)\end{array}$ & \\
\hline 2007 & $\begin{array}{l}\text { Municipalidad } \\
18(3-19)\end{array}$ & $\begin{array}{l}\text { Municipalidad } \\
52.993(0 \text { a 52.993) }\end{array}$ & $\begin{array}{l}\text { CONAF } \\
13.2 \%(22.71 \%)\end{array}$ \\
\hline 2010 & $\begin{array}{l}\text { Municipalidad } \\
\text { 10 (0-10) }\end{array}$ & $\begin{array}{l}\text { Municipalidad } \\
127.667(0 \text { a 127.667) }\end{array}$ & $\begin{array}{l}\text { Agrupación } \\
\text { Maitenes } \\
0 \%(42.05 \%)\end{array}$ \\
\hline
\end{tabular}

Fuente: elaboración propia.

En general, es el gobierno local quien concentra mayor poder dentro de la Red de trabajo de la Mesa de Turismo, debido a la centralidad en que se encuentra dentro del conjunto de actores, canalizando la información entre los diferentes nodos y cooperando en las diversas instancias desarrolladas antes, durante y después de la intervención del Programa Emprende.

\section{Sub grupos.}

Su análisis permite estudiar y conocer el comportamiento de la Red en su conjunto, en función de la posición de los actores en dichas sub estructuras. En este estudio se han considerado dos factores de análisis: Cliqués, que indica al actor o actores que forman el centro neurálgico de la Red, permite conocer la cohesión entre los nodos y el grado de solidaridad que existe entre ellos; y Lambda Set, que muestra a aquellos actores que, si se desconectaran, afectarían al flujo de comunicación entre los nodos de la Red. 
En el cuadro $\mathrm{N}^{\circ} 7$, se observa que el centro neurálgico de la Red (Cliqués) en torno al intercambio de información, está compuesto principalmente por servicios públicos, destacando el binomio que se genera entre la Municipalidad y SERCOTEC (2005), Municipalidad y SENCE (2007) y Municipalidad y SERNATUR (2010).

Para el caso del intercambio de cooperación, el cuadro $\mathrm{N}^{\circ} 8$ muestra que el año 2005 todos los actores de la Mesa de Turismo se encontraban cohesionados, por lo cual se podría asumir que la aplicación del Programa Emprende afectó la relación de trabajo existente hasta ese momento, potenciando a ciertos actores por sobre un trabajo más solidario entre los mismos. En el ámbito de la cooperación, la Municipalidad de Panguipulli sigue cumpliendo un rol protagónico en la instancia de trabajo analizada.

En relación al análisis de Lambda Set o fragmentación de la Red, se observa nuevamente, tanto en el análisis de intercambio de información (cuadro $\mathrm{N}^{\circ} 7$ ) como de cooperación (cuadro $\mathrm{N}^{\circ} 8$ ), que la Municipalidad cumple un rol importante, ya que según este análisis su ausencia afectaría las conexiones generadas dentro de la Red.

Cuadro $N^{\circ} 7$. Indicadores de Sub grupos en torno al intercambio de información.

\begin{tabular}{|c|l|l|}
\hline Subgrupos & Cliqués & Lambda Set \\
\hline 2005 & Municipalidad/SERCOTEC & Municipalidad/SERCOTEC \\
\hline 2007 & Municipalidad/SENCE & $\begin{array}{l}\text { Municipalidad/SERCOTEC/ } \\
\text { SERNATUR/SENCE }\end{array}$ \\
\hline 2010 & Municipalidad/SERNATUR & Municipalidad/SERNATUR/SENCE \\
\hline
\end{tabular}

Fuente: elaboración propia.

Cuadro $\mathrm{N}^{\circ} 8$. Indicadores de Sub grupos en torno al intercambio de cooperación.

\begin{tabular}{|c|l|l|}
\hline Subgrupos & Cliqués & Lambda Set \\
\hline 2005 & Todos los actores & Municipalidad/SERCOTEC \\
\hline 2007 & Municipalidad/SENCE & Municipalidad/SENCE \\
\hline 2010 & $\begin{array}{l}\text { Municipalidad/Pesca } \\
\text { Choshuenco/ } \\
\text { SERNAPESCA }\end{array}$ & Municipalidad/SERNATUR \\
\hline
\end{tabular}

Fuente: elaboración propia.

Del análisis de los sub grupos, se concluye que el nodo Municipalidad es el actor de conexión de los diversos actores en la Red de trabajo tanto para el intercambio de información entre los agentes participantes como al momento de recibir y entregar 
cooperación a los mismos. Su presencia y participación durante el proceso de desarrollo del Programa Emprende permitió fortalecer los flujos internos de la Red y reducir la fragmentación de la Mesa de trabajo.

\section{CONCLUSIONES}

En este trabajo se han analizado las características de las relaciones que se establecen entre los actores públicos y privados que intervienen en la actividad turística, a través de la aplicación de una política publica en un territorio definido, lo que ha permitido identificar los efectos en la dinámica de relación de los actores participantes de la Mesa de turismo de la comuna de Panguipulli.

- Producto de la aplicación del Programa Emprende, diversos servicios públicos debieron reorientar sus instrumentos y líneas de trabajo hacia el territorio comunal, específicamente a la actividad turística. Si bien esta intervención permitió la participación de un mayor número de actores privados en la mesa de trabajo, esta pudiera ser vista como una señal de dependencia por parte de las organizaciones privadas, respecto a los instrumentos de fomento de los servicios públicos. Esto es posible observar en la baja cantidad de actores, antes de comenzar a desarrollarse el Programa en la comuna y una vez finalizado el mismo.

- Se constata a través de este estudio que las interacciones entre el sector público y los actores privados son un factor clave a tener en cuenta en la valoración de la capacidad de acción de cualquier territorio en el ámbito turístico, en especial cuando se realizan a través de programas con una temporalidad de ejecución. En este sentido cobra importancia entonces, la generación de instancias territoriales que sean capaces de empoderarse de las políticas públicas, de tal manera que las intervenciones en los territorios, cumplan con las necesidades requeridas por los actores locales y no se transformen en meras intervenciones más bien "de índole político". Los programas de carácter productivo, deben necesariamente ir acompañados de un trabajo de fortalecimiento de la Red de actores, esto con la finalidad de que, una vez se concluya la intervención, no se afecten las confianzas y el trabajo público-privado. Las políticas públicas sectoriales por si solas no tendrán un buen resultado al momento de construir encadenamientos de aprendizaje interactivo si no se consideran dentro de los objetivos, instancias que fortalezcan a las Redes de trabajo local, las que pudieran verse afectadas o mejor aún fortalecidas, una vez finalizadas las intervenciones.

- Resulta clave reconocer el rol que cumple el gobierno local al momento de potenciar el trabajo público-privado en los territorios, ya que muchas veces es allí donde se concentra el mayor poder de aglutinamiento entre los diversos actores, producto de las confianzas depositadas en los Municipios, en especial por parte de las organizaciones de base 
locales. Los Municipio son actores que quedan finalmente en los territorios y trascienden a cualquier intervención o política estatal.

- Se concluye que, en algunos casos, el sector privado asume una actitud más bien pasiva respecto al desarrollo del Programa Emprende, asociada a una lógica benefactora que traen consigo este tipo de políticas públicas. Esto queda de manifiesto al analizar la disminución en el número de actores y de conexiones antes de la intervención estatal y posterior a su cierre.

- Es necesario potenciar la masa crítica local en relación a las intervenciones que realiza el Estado, permitiendo la apertura de espacios de discusión, para lograr de manera efectiva cumplir con las necesidades que el territorio requiere.

- A nivel de país, existen pocas experiencias de evaluación ex-ante y ex-post de políticas públicas. Las evaluaciones se limitan más bien al ámbito presupuestario. Este estudio permite analizar el ámbito de la generación o no de Redes entre el Estado y la sociedad civil, como un producto paralelo a los objetivos que perseguía el Programa Emprende, por lo cual no es posible concluir si se lograron todos los objetivos que se pretendía alcanzar con la aplicación de esta política pública.

Los resultados expuestos abren un interesante espacio para analizar intervenciones públicas de diverso carácter y en diversas instancias de trabajo, ya que permitiría reorientar los objetivos de las políticas y obtener mejores resultados en su aplicación.

\section{BIBLIOGRÁFIA.}

Aguilar, A. y Lima, F. (2009): ¿Qué son y para qué sirven las Políticas Públicas?, en Contribuciones a las Ciencias Sociales.

Boisier, S. (2003): ¿Y si el desarrollo fuese una emergencia sistémica? Diplomado en Desarrollo, Gobernabilidad y Territorio.

Chile Emprende (2005) La situación de la Micro y Pequeña Empresa en Chile.

De la Maza, G. (2010): Construcción democrática, participación ciudadana y Políticas Públicas en Chile.

De la Maza, G. (2004): Políticas Públicas y Sociedad Civil en Chile: el caso de las Políticas Sociales (1990-2004). Revista Política, No 043, Universidad de Chile.

Ferreiro, A. y Silva, F. (2009): Agencia de Calidad de las Políticas Públicas. Consorcio para la Reforma del Estado.

Fukuyama, F. (2004): La construcción del Estado. Hacia un nuevo orden mundial en el siglo XXI. Ediciones B, Barcelona, España. 
Lahera, E. (2004): Política y Políticas Públicas. División de Desarrollo social.

Lozares, C. (2006): La teoría de redes sociales. Papers, Revista de sociología, 1995, (48) $103-126$

Orellana, C. (2006): Desarrollo Territorial y Empleo. La experiencia de Chile Emprende. SERCOTEC.

Podestá, J. (2001): Problematización de las políticas públicas desde la óptica regional. Última década. Revista $\mathrm{N}^{\circ} 15$ del CIDPA, Viña del Mar. pp 163-175.

Porras, J. y Espinoza, V. (2005): Redes. Enfoques y aplicaciones del análisis de redes sociales (ARS), Universidad Bolivariana, Santiago de Chile.

Sepúlveda, C. (2001): La Economía del Turismo en Chile. Políticas públicas y Recursos Humanos. Proyecto Formación Técnica y Profesional en América Latina, implementada por la CEPAL y la Sociedad Alemana de Cooperación Técnica (GTZ)

Williamson, O. (1985). Las instituciones económicas del Capitalismo: las empresas, mercados, la contratación relacional. Nueva York: The Free Press.

Recibido: 04/05/2012

Aceptado: 18/06/2012

Arbitrado anónimamente 